\title{
Sema4A induces cell morphological changes through B-type plexin-mediated signaling
}

\author{
KAZUNORI YUKAWA $^{1}$, TETSUJI TANAKA ${ }^{2}$, KENJI YOSHIDA $^{1}$, NORIKO TAKEUCHI ${ }^{1}$, \\ TAKUJI ITO $^{1}$, HYOTA TAKAMATSU ${ }^{3}$, HITOSHI KIKUTANI ${ }^{4}$ and ATSUSHI KUMANOGOH ${ }^{3}$ \\ ${ }^{1}$ Department of Physiology, Faculty of Pharmacy, Meijo University, 150 Yagotoyama, Tempaku-ku, \\ Nagoya 468-8503; ${ }^{2}$ Department of Obstetrics and Gynecology, Wakayama Medical University, \\ 811-1 Kimiidera, Wakayama 641-8509; Department of ${ }^{3}$ Immunopathology; \\ ${ }^{4}$ Molecular Immunology, Research Institute for Microbial Diseases, \\ Osaka University, 3-1 Yamada-oka, Suita, Osaka 565-0871, Japan
}

Received September 14, 2009; Accepted November 6, 2009

DOI: $10.3892 /$ ijmm_00000334

\begin{abstract}
Semaphorins are a family of secreted and membranebound proteins known as axonal pathfinders. Sema4A, a member of class 4 semaphorins, induces growth cone collapse of hippocampal neurons. The binding of Sema4A to growth cones indicates the presence of receptors transmitting signals through the intracellular effectors to induce growth cone collapse in hippocampal neurons. Transfection experiments of the candidate receptor genes into COS-7 cells demonstrated that Sema4A binds to axonal guidance receptors Plexin-B1, -B2 and -B3. To identify the functional Sema4A receptor and the signal transduction machinery, COS-7 cell contraction assay was performed, in which intracellular signal transmission induced by Sema4A triggered cell contraction. Expression vectors encoding plexins and Rnd1, a Rho family GTPase, were transfected into COS-7 cells, and a proportion of contracted cells among the transfectants was determined after incubation with Sema4A. The results demonstrated that the combination of Rnd1 and Plexin-B1, -B2 or -B3 induced significant cell contraction, indicating that B-type plexins transmit an intracellular signal of Sema4A through Rnd1. To further study the mechanism of B-type plexin-mediated signaling in Sema4A-induced growth cone collapse, mouse hippocampal neurons transfected with a control or expression plasmid encoding a constitutively active mutant of R-Ras (R-RasQL) were stimulated with Sema4A, followed by the assessment of growth cone collapse. Expression of R-RasQL significantly blocked Sema4Ainduced growth cone collapse in the hippocampal neurons
\end{abstract}

Correspondence to: Dr Kazunori Yukawa, Department of Physiology, Faculty of Pharmacy, Meijo University, 150 Yagotoyama, Tempaku-ku, Nagoya 468-8503, Japan

E-mail:kyukawa@ccmfs.meijo-u.ac.jp

Key words: semaphorin, axon guidance, growth cone collapse, small guanosine triphosphatase, cell contraction, R-Ras compared with the control plasmid. Sema4A thus induces growth cone collapse through the down-regulation of R-Ras activity in mouse hippocampal neurons.

\section{Introduction}

Semaphorins compose a large family of phylogenetically conserved soluble and transmembrane molecules, which are further divided into eight classes (1). Semaphorins were originally identified as repulsive axonal guidance cues which induce growth cone collapse in developing neurons (2-4). Later studies have revealed their widespread roles in a variety of developmental and pathological conditions $(5,6)$. Sema4D, a member of class 4 semaphorins, has facilitated our understanding of how semaphorins regulate axonal repulsion through cytoskeletal changes (7-10). Sema4D, also known as CD100, induces repulsive changes including growth cone collapse in cultured hippocampal neurons and retinal ganglion cells (7). For the repulsion, Sema4D binds to Plexin-B1, a member of the plexins that are the predominant family of semaphorin receptors (7). Both Sema4D and Plexin-B1 contain a distinctively conserved $\sim 400$ amino acid Sema domain that features a seven-blade $\beta$-propeller fold in their extracellular domain $(1,3)$ and they interact with each other through their respective Sema domain (5). In the intracellular region at the $\mathrm{C}$ terminus, Plexin-B1 has two GTPase activating protein (GAP) domains separated by a GTPase binding domain and a PDZ-binding site $(5,7,8)$. Binding of Sema4D to Plexin-B1 induces clustering of Plexin-B1 leading to the activation of the GAP domains, which is facilitated by an active GTPase Rnd1-dependent relief of the GAP domain interaction (8). The GAP activity of Plexin-B1 promotes the conversion of GTP-bound (active) R-Ras to GDP-bound (inactive) R-Ras, resulting in the downregulation of R-Ras activity. This in turn downregulates integrin-based cell adhesion to the extracellular matrix, thus leading to growth cone collapse in cultured hippocampal neurons (8). Plexin-B1 also allows intracellular RhoA-specific guanine nucleotide exchange factors (GEFs), PDZ-RhoGEF and leukemia-associated RhoGEF (LARG), to bind to the PDZ-binding motif at the 
C terminus of Plexin-B1 (7). Sema4D binding to Plexin-B1 stimulates the GEF activities of PDZ-RhoGEF and LARG, promoting the conversion of the GDP-bound form of RhoA, a member of Rho GTPases crucial for the regulation of actin and microtubule dynamics (7), to the GTP-bound form. The increase in the GTP-bound forms of RhoA enhances actomyosin contractility through Rho kinase activation and myosin light chain phosphorylation, thereby leading to growth cone collapse of hippocampal neurons $(5,7)$. Both the R-Ras GAP activity and PDZ-RhoGEF-mediated RhoA activation by plexin-B1 are necessary for the Sema4D-induced growth cone collapse in cultured hippocampal neurons $(7,8)$.

In mouse, the expression of Sema4A, another class 4 semaphorin originally identified as semB, gradually increases during embryonic development (11) and becomes prominent in the adult brain, spleen, lung, kidney, testis and mammary gland (12). Sema4A expressed in dendritic cells and B-cells enhances the activation of T-cells and the generation of antigenspecific T-cells by virtue of the interaction with the Sema4A receptor, Tim-2, a member of the T-cell immunoglobulin domain and mucin domain (Tim) proteins expressed on activated T-cells (12). Our previous study showed that recombinant Sema4A induces growth cone collapse in mouse hippocampal neurons (13). The binding of Sema4A to the growth cones indicated the presence of a specific receptor transmitting Sema4A signal via the intracellular effectors to induce growth cone collapse in the neurons (13). In the current study, to identify the functional Sema4A receptor and the signal transduction machinery, we first performed COS-7 cell contraction assay, in which Sema4A-induced intracellular signaling triggered cell contraction (14). The assay was performed to examine whether one of the B-type plexins (Plexin-B1, -B2 or -B3) and Rnd1, a small GTP-binding protein of the Rho family, constituted a signal transduction machinery of Sema4A. Using primary hippocampal neurons, we also investigated whether the intrinsic guanosine triphosphatase (GTPase) activity of R-Ras, a member of the Ras superfamily of small GTP-binding proteins, is regulated in Sema4A-induced growth cone collapse in mouse hippocampal neurons.

\section{Materials and methods}

Expression plasmids and antibodies. Expression plasmids encoding hemagglutinin (HA)- and GFP-tagged Rnd1, Myctagged Plexin-B1, -C1 and HA-tagged R-Ras-QL (Q87L) were generously provided by Dr M. Negishi, Kyoto University, Japan (8). Expression plasmids encoding VSV-G-tagged Plexin-B2, -B3 and -D1 were generous gifts from Dr L. Tamagnone, University of Torino, Italy. The following antibodies were used in this study: mouse monoclonal antibody against Myc (Santa Cruz Biotechnology, Santa Cruz, CA), a rat monoclonal antibody against HA (Roche, Mannheim, Germany), a mouse monoclonal antibody against VSV-G (Roche), a mouse monoclonal antibody against GFP conjugated to Alexa 594 (Molecular Probes, Eugene, OR), and secondary antibodies conjugated to Alexa 594 (Molecular Probes).

Production of Sema4A-Fc. Recombinant soluble mouse Sema4A protein comprising the putative extracellular region fused to the human immunoglobulin- $\gamma 1$ (IgG1) Fc fragment (Sema4A-Fc) was prepared as previously described (12).

Cell culture and transfection. COS-7 cells were cultured in Dulbecco's modified Eagle's medium containing $10 \%$ fetal calf serum, $4 \mathrm{mM}$ glutamine, 100 units $/ \mathrm{ml}$ penicillin, and $0.2 \mathrm{mg} / \mathrm{ml}$ streptomycin at $37^{\circ} \mathrm{C}$ in a humidified $5 \% \mathrm{CO}_{2} / 95 \%$ air incubator. COS-7 cells were transiently transfected with one or a combination of expression plasmids in lipofectamine 2000 (Invitrogen, Carlsbad, CA) according to the manufacturer's instructions. Primary hippocampal neurons were isolated from embryos from pregnant mice at embryonic day 17 and cultured on glass coverslips in 24-well plates as described previously $(15,16)$ except for the use of the Sumilon Nerve-Cell Culture System (Sumitomo Bakelite, Tokyo, Japan). Primary hippocampal neurons on glass coverslips were transfected with Lipofectamine 2000 containing expression plasmids after $12 \mathrm{~h}$ in culture.

Growth cone collapse. Hippocampal neurons cultured from E17 mouse embryos for 1.5 days were treated with Sema4A-Fc $(10 \mu \mathrm{g} / \mathrm{ml})$ or its vehicle, for $1 \mathrm{~h}$ at $37^{\circ} \mathrm{C}$. Neurons were fixed with $4 \%$ paraformaldehyde and processed for immunocytochemistry. FITC-labeled Phalloidin (Sigma, St. Louis, MO, USA) was used to detect actin filaments in growth cones. Neurons were scored for the presence of growth cones. Growth cones with round-tipped or pencil-like appearance represented the collapsed form $(17,18)$. Data were expressed as a percentage of collapsed growth cones out of the total number of growth cones, and the mean values were calculated from four separate experiments.

COS-7 cell binding and contraction assay. COS-7 cells ( $1 \times 10^{4}$ cells) were seeded onto round $10-\mathrm{mm}$ glass coverslips coated with lysine/laminin and transfected with expression plasmids encoding mouse and human Plexin-B1, -B2, -B3, -C1, -D1, Tim-2 and/or Rnd1. After transfection (24 h), COS-7 cells were incubated with recombinant Sema4A fused with heat stable alkaline phosphatase (Sema4A-AP) for $90 \mathrm{~min}$ at room temperature. Cells were postfixed for $30 \mathrm{sec}$ in a buffer containing $60 \%$ acetone, $3 \%$ formaldehyde, and $20 \mathrm{mM}$ HEPES ( $\mathrm{pH} \mathrm{7.5),} \mathrm{followed} \mathrm{by} \mathrm{heating} \mathrm{at} 65^{\circ} \mathrm{C}$ for $20 \mathrm{~min}$ to inactivate endogenous alkaline phosphatase. The binding of Sema4A to COS-7 cells was visualized by reaction with NBT/BCIP (19). For COS-7 cell contraction assay, COS-7 cells transiently transfected with individual plasmids were treated $24 \mathrm{~h}$ after the transfection with Sema4A-Fc $(10 \mu \mathrm{g} / \mathrm{ml})$ or its vehicle for $5 \mathrm{~min}$ at $37^{\circ} \mathrm{C}$. The size of transfected cells was visualized using a fluorescence microscope and their images were captured with a digital camera (HV-C20S; Nikon, Tokyo, Japan).

Statistics. All data represent means \pm SEM. Statistical significance was determined by analysis of variance, and $\mathrm{p}<0.05$ was considered as a minimal level of significance.

\section{Results}

Sema4A binds to B-type plexins. To examine which guidance receptors Sema4A binds to, binding assays with COS-7 cells expressing candidate receptors were performed using 

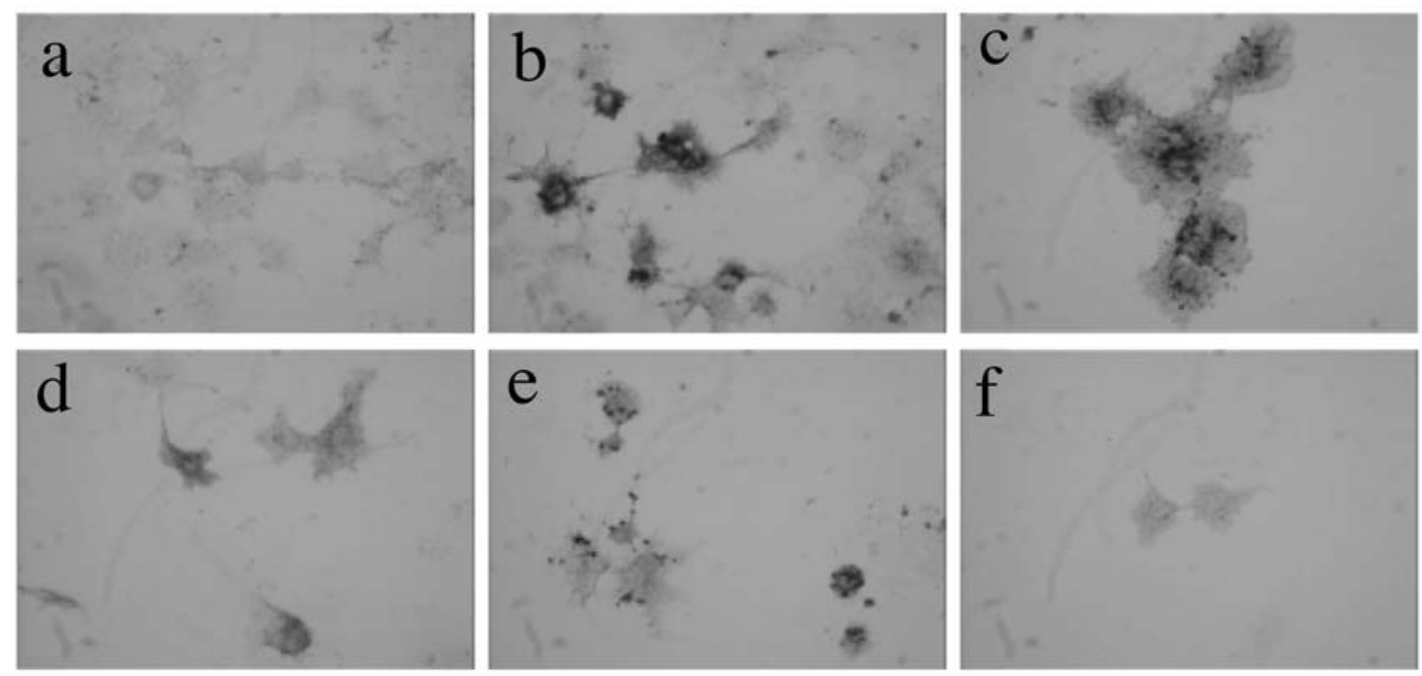

Figure 1. Sema4A binds to Plexin-B1, -B2 and -B3. Sema4A binds to COS-7 cells expressing mouse Plexin-B1 (b), human Plexin-B1 (c), human Plexin-B2 (d), and human Plexin-B3 (e) on lysine/laminin-coated coverslips. COS-7 cells were initially transfected with expression plasmids encoding EGFP (a), mouse Plexin-B1 (b), human Plexin-B1 (c), Plexin-B2 (d), Plexin-B3 (e) and Plexin-C1 (f), and then the binding ability of Sema4A to each plexin was examined with Sema4A-AP.

recombinant Sema4A fused to heat stable alkaline phosphatase. As shown in Fig. 1, the assay revealed the binding of Sema4A to COS-7 cells expressing one of the B-type plexins (Plexin-B1, -B2, or -B3). Recombinant Sema4A did not exhibit significant binding to $\mathrm{COS}-7$ cells expressing either the control vector or Plexin-C1 (Fig. 1). Thus, the binding assays showed that Sema4A interacts specifically with B-type plexins.

Sema4A uses B-type plexins and Rnd family GTPase, Rndl, to induce cell contraction. To identify functional Sema4A receptor and the signal transduction machinery, COS-7 cell contraction assay was performed, in which Sema4A-induced intracellular signal transmission leads to cell contraction (14). Sema4A did not induce any morphological changes in COS-7 cells expressing Plexin-B1 alone (Fig. 2A). However, COS-7 cells expressing both Plexin-B1 and Rnd1 showed significant contraction of the cell body (Fig. 2A). Quantitative analysis of the COS-7 cell contraction was performed to obtain the percentage of cells showing contraction out of the total number of the transfected cells. The results showed that cells coexpressing Plexin-B1 and Rnd1 exhibited significantly higher collapses than cells transfected with the control plasmid (Fig. 2B). The assay also revealed that Sema4A induced significant contraction of COS-7 cells expressing both Plexin-B2 and Rnd1 (Fig. 2B) as well as Plexin-B3 and Rnd1 (Fig. 2B).

Involvement of R-Ras in the Sema4A-induced growth cone collapse. Downregulation of R-Ras activity by a Plexin-B1Rnd1 complex is integral to the growth cone collapse in hippocampal neurons induced by Sema4D, another class 4 semaphorin (8). To examine whether the downregulation of $\mathrm{R}$-Ras activity is also involved in Sema4A-induced growth cone collapse in hippocampal neurons, mouse hippocampal neurons expressing a constitutively active mutant of R-Ras (R-RasQL) were treated with Sema4A, and growth cone morphology was analyzed by immunofluorescence methods.
A
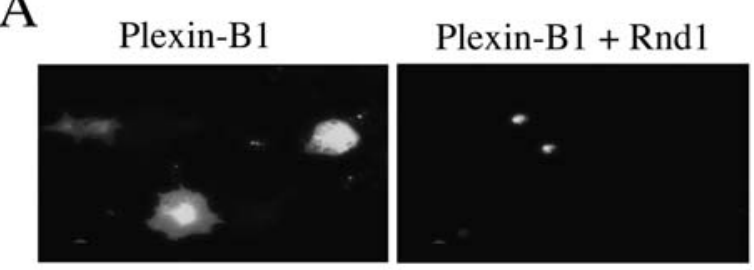

B

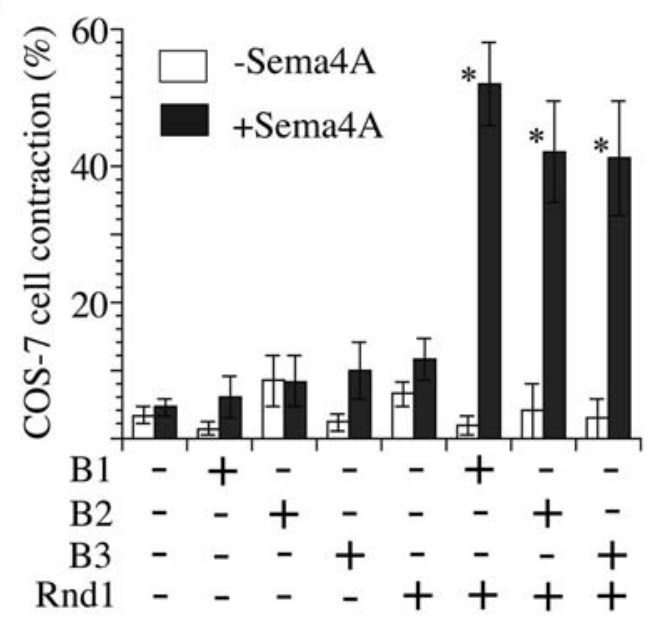

Figure 2. Sema4A-induced COS-7 cell contraction is mediated by B-type plexin and Rnd1. (A) COS-7 cells were transfected with the indicated plasmids and incubated with Sema4A for $5 \mathrm{~min} 24 \mathrm{~h}$ after transfection. The transfected cells were visualized by the fluorescence from the antibodies against GFP, Myc, and HA. Sema4A did not induce contraction of COS-7 cells overexpressing Plexin-B1 alone, whereas Sema4A induced significant contraction of COS-7 cells coexpressing Rnd1 and one of the B-type plexins (Plexin-B1, -B2, or -B3). The scale bar represents $10 \mu \mathrm{m}$. (B) Quantitative analysis of COS-7 cell contraction mediated by Plexin-B1, -B2, -B3, or Rnd1 in response to Sema4A binding. COS-7 cells were transfected with an expression vector encoding HA tag, GFP, Plexin-B1, -B2, -B3, or Rnd1. After transfection $(24 \mathrm{~h})$, cells were incubated with Sema4A for $5 \mathrm{~min}$. Double positive cells with both HA (or GFP) and Myc-staining were counted and COS-7 cell contraction was expressed as a percentage of contracted cells out of the total transfected cells. About 50 cells were assessed in one experiment, and the data represent the mean \pm SEM values of three independent experiments. B1, Plexin-B1; B2, Plexin-B2; B3, Plexin-B3. 


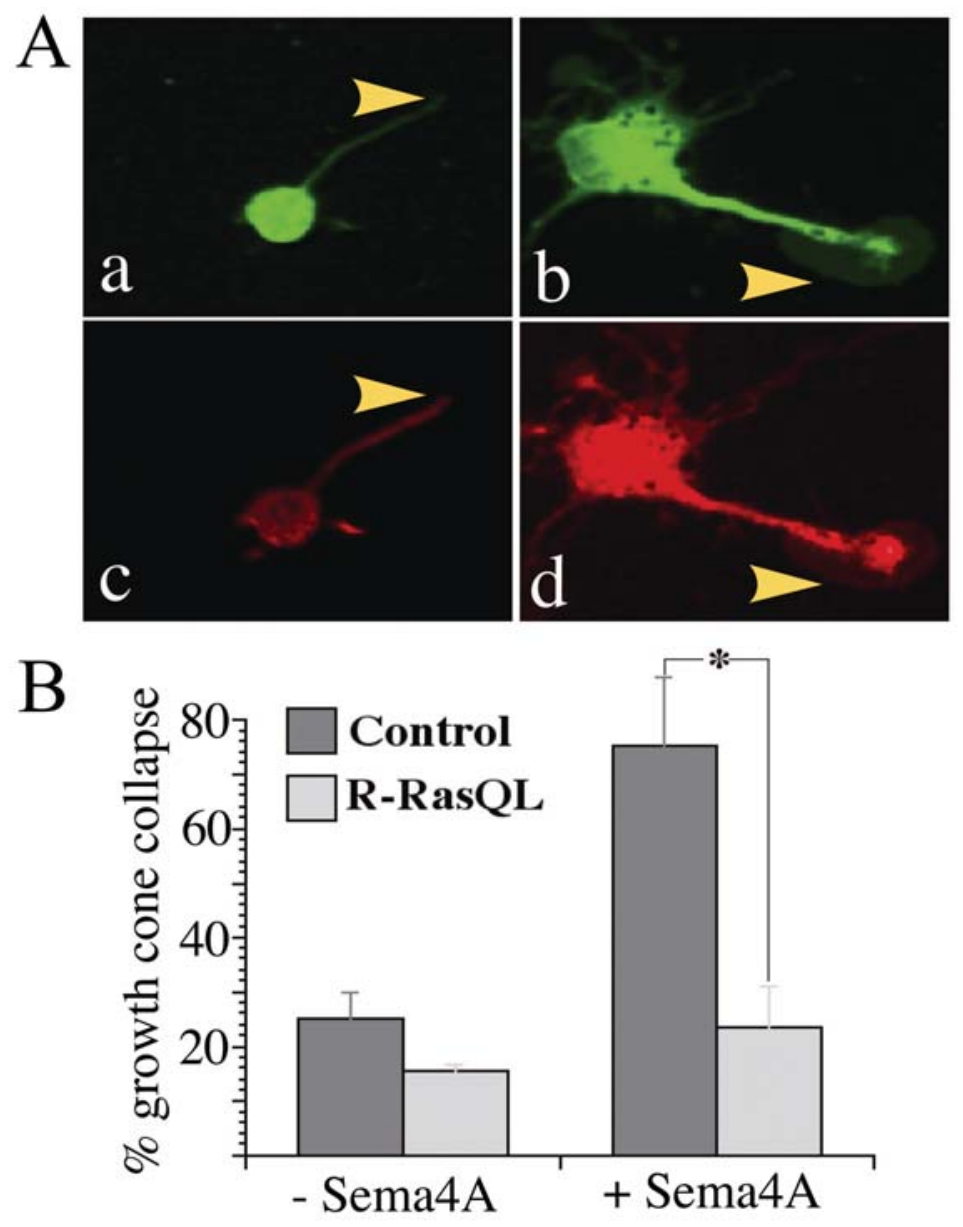

Figure 3. Downregulation of R-Ras activity is required for the Sema4A-induced growth cone collapse in mouse hippocampal neurons. (A) Mouse hippocampal neurons transfected with a control or expression plasmid encoding a constitutively active mutant of R-Ras (R-RasQL) were stimulated with Sema4A. Growth cones of the transfectants were shown by the immunofluorescence from the antibody against HA tag. The presence of filopodia and lamellipodia visualized by the binding of Alexa 594-conjugated phalloidin to filamentous actin (F-actin) defined a growth cone. Sema4A induced growth cone collapse in the primary hippocampal neurons expressing the control vector (arrowhead in a and c). In contrast, Sema4A did not induce growth cone collapse in the hippocampal neurons expressing R-RasQL (arrowhead in b and d). (B) Growth cone collapse was scored as a percentage of the transfected neurons with collapsed growth cones out of the total number of transfected cells. Hippocampal neurons with the control vector exhibited significantly higher growth cone collapses in response to Sema4A (+ Sema4A), as compared with hippocampal neurons expressing the control vector or R-RasQL cultured without Sema4A (- Sema4A). Expression of R-RasQL, but not the control plasmid, significantly blocked Sema4A-induced growth cone collapse in mouse hippocampal neurons.

Sema4A induced growth cone collapse in primary hippocampal neurons expressing the control vector (Fig. 3A-a and -c). In contrast, hippocampal neurons expressing R-RasQL did not exhibit growth cone collapse in response to Sema4A (Fig. 3A-b and $-\mathrm{d})$. Quantitative assay measuring the percentage of the transfected neurons with collapsed growth cones out of the total number of the transfected cells clearly showed that the overexpression of R-RasQL significantly blocked the Sema4Ainduced growth cone collapse in the primary hippocampal neurons compared with the overexpression of the control plasmid (Fig. 3B).

\section{Discussion}

Our molecular and cellular analyses have revealed that Sema4A induces B-type plexin-mediated collapse of COS-7 cells through Rnd1 and growth cone collapse of mouse hippocampal neurons through the downregulation of R-Ras activity. Our findings suggest the crucial involvements of Sema4A in the neurodevelopmental processes via the activation of B-type plexin-mediated signaling.

The binding of Sema4A to B-type plexins in this study is consistent with the recent finding that showed the binding of ${ }^{125}$ I-labeled Sema4A to the membranes of cells expressing one of B-type plexins or Plexin-D1 (20). In the vascular system, Sema4A binds to Plexin-D1 expressed on the endothelial cells and mediates antiangiogenic activity (20). In the immune system, Sema4A binds to Tim-2 on T-cells, leading to the activation of their proliferation and differentiation (12). Induction of Plexin-D1 expression allows Sema4A to reduce cell adhesion to fibronectin and collagen type 4 , but not to poly-D-lysine and laminin (20). Another study reported that Sema4C, another member of class 4 semaphorins, weakly enhanced adhesion of granule cell populations to laminin, but not to poly-L-lysine or fibronectin (21). In our experimental conditions using lysine/laminin coated glass coverslips as matrices, the binding of Sema4A was observed only to B-type plexins but not to either Plexin-C1 or -D1. This may indicate 
that both the binding of semaphorins to plexin receptors and the physiological effects of semaphorin-plexin interaction vary depending on the context generated by the combination of various cell types and extracellular matrices.

In our COS-7 cell contraction assay, Sema4A induced significant contraction of COS-7 cells coexpressing Rnd1 and one of the B-type plexins. Plexin-B1 functions as a molecule with R-Ras GAP activity (8), and other B-type plexins are also believed to have R-Ras GAP activities (5). It is necessary for Rnd1 to bind to plexin-B proteins to induce their R-Ras GAP activity inside cells $(8,22)$. Our experimental results showed that, upon binding to plexin-B proteins expressed on the COS-7 cell membranes, Sema4A signaled cells to induce morphological changes, that is, cell contraction, through Rnd1.

In our experiments to explore if Sema4A exhibits any physiological roles in the directional guidance of axonal elongation during brain development, overexpression of the constitutively active mutant of R-Ras in cultured hippocampal neurons significantly blocked growth cone collapse induced by Sema4A. Sema4D promotes growth cone collapse in hippocampal neurons by binding to plexin-B1. Upon Sema4D binding, Plexin-B1 through the interaction with Rnd1 enhances the GAP activity toward R-Ras, leading to the downregulation of R-Ras/integrin pathway and thus to the decrease in the adhesion of growth cones to extracellular matrices $(8,9,22)$. It is therefore possible that Sema4A also promotes growth cone collapse of cultured hippocampal neurons by stimulating R-Ras GAP activities through B-type plexins. Recent studies investigating the roles of semaphorins revealed not only the crucial involvements of each semaphorin in axon guidance but also in the ligand-receptor interactions between semaphorins and plexins essential for neural development (21-25). Plexin-B2 is not only a crucial component of neural tube development but also a key molecule that directs proliferation and migration of granule cell precursors in the developing dentate gyrus, olfactory bulb, and cerebellum (21). The expression pattern, high affinity and specific nature of Sema4C-Plexin-B2 binding and their functional studies consistently propose Sema4C as a true ligand for Plexin-B2 (21). Plexin-B3 has been reported to interact in vitro with a member of class 5 semaphorins, Sema5A (23). The analysis of Plexin-B1 knockout mice has shown that Plexin-B1 is integral for gonadotropin hormonereleasing hormone-1 (GnRH-1) cells to migrate from olfactory placode to hypothalamic areas (24). Sema4D promotes directional migration in immortalized GnRH-1 neurons by coupling Plexin-B1 with activation of the Met tyrosine kinase (24). However, because of the failure to detect migratory defects of GnRH-1 neurons in Sema4D-deficient mice, the reality of the semaphorin ligand controlling the migration of GnRH-1 cells remains controversial (24). This raises the possibility that many semaphorins including Sema4D and Sema4A function in concert to regulate the migration of GnRH-1 cells in vivo. Analysis of Sema4A-deficient mice revealed the non-redundant roles of Sema4A in the developmental path of retinal photoreceptor cells, although the receptors involved have not yet been clarified (25). For these reasons, Sema4A may play a crucial role in the development of the neural system through the interaction with B-type plexins in concert with other semaphorin members.
In summary, our study showed that the binding of Sema4A to Plexin-B1, -B2 or -B3 in vitro leads to the B-type plexinmediated contraction of COS-7 cells through Rnd1. Our study further demonstrated that Sema4A promotes growth cone collapses of mouse hippocampal neurons through the downregulation of R-Ras activity. Our results therefore suggest the crucial involvement of Sema4A in the growth cone guidance through the activation of B-type plexin-mediated signaling in developing neurons.

\section{Acknowledgements}

We are grateful to K. Kubota, M. Kishino, T. Ueyama and E. Yukawa for their encouragement and support. This study was partly supported by Grants-in-Aid for Scientific Research from the Ministry of Education, Science, Sports and Culture of Japan.

\section{References}

1. Semaphorin Nomenclature Committee: Unified nomenclature for the semaphorins/collapsins. Cell 97: 551-552, 1999.

2. Kapfhammer JP and Raper JA: Collapse of growth cone structure on contact with specific neurites in culture. J Neurosci 7: 201-212, 1987.

3. Nakamura F, Kalb RG and Strittmatter SM: Molecular basis of semaphorin-mediated axon guidance. J Neurobiol 44: 219-229, 2000.

4. Raper JA: Semaphorins and their receptors in vertebrates and invertebrates. Curr Opin Neurobiol 10: 88-94, 2000.

5. Kruger RP, Aurandt J and Guan KL: Semaphorins command cells to move. Nat Rev Mol Cell Biol 6: 789-800, 2005.

6. Tamagnone L and Comoglio PM: To move or not to move? Semaphorin signalling in cell migration. EMBO Rep 5: 356-361, 2004.

7. Swiercz JM, Kuner R, Behrens J and Offermanns S: Plexin-B1 directly interacts with PDZ-RhoGEF/LARG to regulate RhoA and growth cone morphology. Neuron 35: 51-63, 2002.

8. Oinuma I, Ishikawa Y, Katoh H and Negishi M: The semaphorin 4D receptor plexin-B1 is a GTPase activating protein for R-Ras. Science 305: 862-865, 2004.

9. Oinuma I, Katoh H and Negishi M: Semaphorin 4D/Plexin-B1mediated R-Ras GAP activity inhibits cell migration by regulating B1 integrin activity. J Cell Biol 173: 601-613, 2006.

10. Ito Y, Oinuma I, Katoh H, Kaibuchi K and Negishi M: Sema4D/plexin-B1 activates GSK-3ß through R-Ras GAP activity, inducing growth cone collapse. EMBO Rep 7: 704-709, 2006.

11. Püschel AW, Adams RH and Betz H: Murine semaphorin D/ collapsin is a member of a diverse gene family and creates domains inhibitory for axonal extension. Neuron 14: 941-948, 1995.

12. Kumanogoh A, Marukawa S, Suzuki K, Takegahara N, Watanabe C, Ch'ng E, Ishida I, Fujimura H, Sakoda S, Yoshida K and Kikutani H: Class IV semaphorin Sema4A enhances T-cell activation and interacts with Tim-2. Nature 419: 629-633, 2002.

13. Yukawa K, Tanaka T, Bai T, Ueyama T, Owada-Makabe K, Tsubota Y, Maeda M, Suzuki K, Kikutani H and Kumanogoh A: Semaphorin 4A induces growth cone collapse of hippocampal neurons in a Rho/Rho-kinase-dependent manner. Int J Mol Med 16: $115-118,2005$.

14. Turner LJ and Hall A: Plexin-induced collapse assay in COS cells. Methods Enzymol 406: 665-676, 2006.

15. Yukawa K, Tanaka T, Tsuji S and Akira S: Expressions of CCAAT/enhancer-binding proteins $\beta$ and $\delta$ and their activities are intensified by cAMP signaling as well as $\mathrm{Ca}^{2+} /$ calmodulin kinases activation in hippocampal neurons. J Biol Chem 273: 31345-31351, 1998.

16. Yukawa K, Tanaka T, Tsuji S and Akira S: Regulation of transcription factor C/ATF by the cAMP signal activation in hippocampal neurons, and molecular interaction of C/ATF with signal integrator $\mathrm{CBP} / \mathrm{p} 300$. Mol Brain Res 69: 124-134, 1999. 
17. Luo Y, Raible D and Raper JA: Collapsin: a protein in brain that induce the collapse and paralysis of neuronal growth cones. Cell 75: 217-228, 1993.

18. Fan J, Mansfield SG, Redmond T, Gordon-Weeks PR and Raper JA: The organization of F-actin and microtubules in growth cones exposed to a brain-derived collapsing factor. J Cell Biol 121: 867-878, 1993.

19. Flanagan JG and Leder P: The kit ligand: a cell surface molecule altered in steel mutant fibroblasts. Cell 63: 185-194, 1990.

20. Toyofuku T, Yabuki M, Kamei J, Kamei M, Makino N, Kumanogoh A and Hori M: Semaphorin-4A, an activator for T-cell-mediated immunity, suppresses angiogenesis via Plexin-D1. EMBO J 26: 1373-1384, 2007.

21. Deng S, Hirschberg A, Worzfeld T, Penachioni JY, Korostylev A, Swiercz JM, Vodrazka P, Mauti O, Stoeckli ET, Tamagnone L, Offermanns S and Kuner R: Plexin-B2, but not Plexin-B1, critically modulates neuronal migration and patterning of the developing nervous system in vivo. J Neurosci 27:6333-6347, 2007
22. Oinuma I, Katoh $\mathrm{H}$ and Negishi M: Molecular dissection of the semaphorin 4D receptor Plexin-B1-stimulated R-Ras GTPaseactivating protein activity and neurite remodeling in hippocampal neurons. J Neurosci 24: 11473-11480, 2004.

23. Artigiani S, Conrotto P, Fazzari P, Gilestro GF, Barberis D, Giordano S, Comoglio PM and Tamagnone L: Plexin-B3 is a functional receptor for semaphorin 5A. EMBO Rep 5: 710-714, 2004.

24. Giacobini P, Messina A, Morello F, Ferraris N, Corso S, Penachioni J, Giordano S, Tamagnone L and Fasolo A: Semaphorin 4D regulates gonadotropin hormone-releasing hormone-1 neuronal migration through PlexinB1-Met complex. J Cell Biol 183: 555-566, 2008.

25. Rice DS, Huang W, Jones HA, Hansen G, Ye GL, Xu N Wilson EA, Troughton K, Vaddi K, Newton RC, Zambrowicz BP and Sands AT: Severe retinal degeneration associated with disruption of semaphorin 4A. Invest Ophthalmol Vis Sci 45: 2767-2777, 2004 